\title{
ANÁLISE ESPECTRAL DO VENTO NO CENTRO DE LANÇAMENTO DE ALCÂNTARA(CLA)
}

\author{
ROBERTO MAGNAGO ${ }^{1}$, GILBERTO FISCH${ }^{1}$, OSVALDO MORAES ${ }^{2}$
}

\author{
${ }^{1}$ Instituto de Aeronáutica e Espaço, Divisão de Ciências Atmosféricas (IAE/ACA), São José dos Campos. SP. \\ ${ }^{2}$ Universidade Federal de Santa Maria (UFSM), Santa Maria, RS \\ roberto_magnago@yahoo.com.br; gfisch@iae.cta.br; osvaldo.moraes@pesquisador.cnpq.br
}

Recebido Dezembro 2008 - Aceito Outubro 2009

\begin{abstract}
RESUMO
Neste trabalho analisam-se as características espectrais da turbulência atmosférica na região do Centro de Lançamento de Alcântara (CLA), que sofre a influência de uma falésia e da brisa marítima provinda do oceano, visando obter informações sobre a estrutura turbulenta do local. Determinaram-se os espectros das 3 componentes do vento $(u, v$ e $w)$ na camada limite superficial sobre esse terreno homogêneo, visando aplicações no Projeto e Desenvolvimento dos foguetes de sondagem e do Veículo Lançador de Satélite (VLS) realizado pelo IAE. Os dados foram coletados no mês de agosto do ano de 1999 através de um anemômetro sônico tridimensional. Também se obteve resultados envolvendo as relações $\sigma_{u} / u_{*}, \sigma_{v} / u_{*}$ e $\sigma_{w} / u_{*}$, todos condizentes com os previstos na literatura.

Palavras Chaves: Espectro turbulento do vento, falésia, terrenos planos.
\end{abstract}

\begin{abstract}
SPECTRALANALYSIS OF THE SURFACE WINDS AT THE ALCÂNTARA LAUCHING CENTER (CLA)

In this work is analyzed the spectral characteristics of atmospheric turbulence in the Alcântara Launching Center (CLA), which suffers the influence of a cliff and of the sea breeze coming from the ocean. The atmospheric spectra were determined for the 3 wind components $(u, v$, and $w$ ) in the superficial boundary layer, to be used for the Research and Developpment (R\&D) of sounding rockets and Satellite Launch Vehicle (VLS) build by IAE. The data were collected during the month of August, 1999 using a 3D sonic anemometer. Results involving the $\sigma_{u} / u_{*}$, $\sigma_{v} / u_{*}$ and $\sigma_{w} / u_{*}$ relations are obtained, and are all consistent with those reported in literature.
\end{abstract}

Keywords: Wind turbulent spectra, coastal cliff, flat terrain.

\section{INTRODUÇÃO}

A camada limite superficial é a parte baixa da camada limite atmosférica onde existe a troca de energia, de vapor d'água e de momentum, entre a superfície terrestre e a atmosfera, sendo esta troca a responsável pelas características dinâmicas e termodinâmicas do escoamento. O mais importante efeito desta interação é a geração de movimentos turbulentos, desenvolvidos em várias escalas no tempo e no espaço (Nieuwtadt e Van Dop, 1981).

Devido a diversos experimentos de campo realizados nas últimas décadas, a estrutura da turbulência sobre superfícies homogêneas e sob várias condições atmosféricas é razoavelmente bem entendida. Experimentos clássicos como o de Kansas em 1968 (Lee et al., 2004), primeiro experimento de campo a utilizar sensores com alta freqüência de coleta e pioneiro na análise dos dados em tempo real, de Minnesota em 1979 e de Cabauw em 1984, ajudaram nesse entendimento. Análises dessa estrutura levaram ao aprimoramento de Teorias de Similaridades que descrevem bem os fluxos turbulentos sobre terrenos homogêneos. No Brasil ainda são poucos os estudos que determinaram a análise espectral, ressaltando-se o estudo realizado por Moraes (2000) para a região plana dos pampas gaúchos e por Magnago et al. (2009) para uma região de topografia complexa.

A região de estudo deste trabalho abrange o Centro de Lançamento Alcântara (CLA), que é o portal brasileiro para o espaço. Uma compreensão do regime de ventos e da turbulência atmosférica na camada limite superficial é de grande importância nos estudos da Meteorologia Aeroespacial, 
pois de suas características são extraídas informações básicas para o P\&D de foguetes de sondagem e Veículos Lançadores de Satélite (VLS). Os foguetes são projetados e construídos para suportarem uma determinada carga pela ação do vento na superfície, além do fato de que a sua trajetória, controle e guiagem são determinados pelo perfil do vento. Diversos estudos sobre as características turbulentas do local já foram realizados, quer seja por observações (Fisch, 1999 e Roballo e Fisch, 2008), quer seja por ensaios em túnel de vento (Roballo e Fisch, 2008 e Pires et al., 2009). Também já foram realizados estudos sobre a atuação de sistemas atmosféricos no local (Barbosa et al., 2006, Coutinho e Fisch, 2007), entre outros.

As características do espectro da velocidade na camada limite atmosférica sobre terrenos homogêneos têm surgido de estudos observacionais conduzidos em vários lugares. Já foi observado que o espectro segue a Teoria de Similaridade de Monin-Obukhov dentro da camada superficial (por exemplo, Moraes 2000). As características espectrais nas altas freqüências são consistentes com a isotropia local e no sub-intervalo inercial o espectro decai com a razão $n^{-5 / 3}$, sendo que $n$ é a freqüência. Kaimal et al. (1972) em seu artigo clássico mostrou que o espectro de freqüência do vento, quando plotados em função de parâmetros adimensionais da camada superficial são reduzidos a uma família de curvas universais, que são funções somente de $z$ $/ L$ nas baixas freqüências, mas convergem para uma única curva universal no sub-intervalo inercial. As exceções são as regiões de entrada de energia dos espectros das componentes de velocidades horizontais, $u$ e $v$, na camada instável. Estas evidências mostram, que os espectros das velocidades horizontais sobre condições convectivas seguem leis de similaridade diferentes nas várias regiões do espectro na camada superficial.

O objetivo principal deste trabalho é analisar as características espectrais da turbulência atmosférica na região do CLA, que sofre a influência de uma falésia e da brisa marítimica provinda do oceano, visando obter informações sobre a estrutura turbulenta do local. Também é determinado o espectro de freqüência das componentes do vento na camada limite superficial, sobre esse terreno homogêneo, visando aplicações no Projeto e Desenvolvimento dos foguetes de sondagem e do Veículo Lançador de Satélite (VLS) realizado pelo IAE.

\section{CARACTERÍSTICAS ESPECTRAIS DA TURBULÊNCIA}

Escoamentos turbulentos como aqueles que ocorrem na camada limite atmosférica, podem ser vistos como uma superposição de turbilhões - padrões coerentes de velocidade, vorticidade e pressão - com vários tipos de tamanhos. Estes turbilhões interagem continuamente com o escoamento médio, a partir do qual eles derivam sua energia, e também interagem entre si. Os grandes turbilhões contêm a maior parte da energia cinética e são os responsáveis pela maioria do transporte na turbulência, através das instabilidades no escoamento de fundo. O forçante aleatório que provoca essas instabilidades é fornecido pela turbulência existente. Este processo é bem representado nos termos de produção da equação da Energia Cinética Turbulenta descrita or:

$$
\begin{aligned}
& \frac{\bar{D} \bar{e}}{D t}=\frac{\partial \bar{e}}{\partial t}=-\left(\overline{u^{\prime} w^{\prime}}\right)\left(\frac{\partial \bar{u}}{\partial z}\right)+\frac{g}{\bar{\theta}}\left(\overline{w^{\prime} \theta^{\prime}}\right)-\frac{1}{\rho} \frac{\partial}{\partial z}\left(\overline{w^{\prime} p^{\prime}}\right)- \\
& \frac{\partial}{\partial z}\left(\overline{e w^{\prime}}\right)-\varepsilon
\end{aligned}
$$

sendo que $e=(1 / 2)\left(u^{, 2}+v^{, 2}+w^{, 2}\right)$, $p$ é a pressão atmosférica, e é a razão de dissipação da energia cinética turbulenta.

A energia contida nos turbilhões está também sujeita a instabilidades, as quais, neste caso, seriam provocadas por outros turbilhões. Isto impõe a estes turbilhões um tempo de vida finito e ele se quebra em turbilhões menores. Este processo se repete em todas as escalas até os turbilhões tornarem-se suficientemente pequenos, e sobre as quais a viscosidade os afeta diretamente, convertendo sua energia cinética em energia interna na forma de calor. A ação da viscosidade é representada no termo de dissipação da equação da energia cinética turbulenta.

Para entender a conversão de energia cinética média em energia cinética turbulenta, junto com a transferência desta energia dos turbilhões maiores para os turbilhões de escalas menores num processo denominado de “cascata”, e esta última conversão dos turbilhões menores para energia em forma de calor por viscosidade, deve-se isolar as diferentes escalas do movimento turbulento e analisar separadamente seu comportamento. O uso do espectro de Fourier da turbulência é uma forma coerente de fazer isso. A representação espectral associada com cada escala de movimento, energia cinética e variância ou fluxo turbulento, contribuem para o todo e dá uma nova perspectiva sobre a estrutura da camada limite planetária.

O espectro das flutuações na camada limite cobre uma escala que abrange desde milímetros a quilômetros na escala espacial e de frações de segundo a hora nas escalas temporais.

\subsection{Espectro de Energia}

O espectro mede a distribuição das variâncias de uma variável sobre comprimento de onda ou freqüência. Se a variável é uma componente de velocidade, o espectro descreve a distribuição da energia cinética sobre o comprimento de onda 
ou freqüência. Uma visualização conceitual útil da distribuição de energia no espaço de número de ondas, quando a turbulência é homogênea em todas as direções, é gerado pelo espectro de energia do escalar $E(k)$, onde $E(k)$ representa a contribuição para a energia cinética total dos modos de Fourier com magnitude de número de onda entre $k$ e $k+\mathrm{d} k$ sendo $k=|\vec{k}|$.

Na Figura 1 pode-se identificar três regiões espectrais principais, referentes ao fluxo turbulento da camada limite. $\mathrm{Na}$ Figura 1 a região $A$ representa a região de entrada da energia turbulenta produzida pelo empuxo térmico e/ou cisalhamento mecânico. A região $B$ é denominada subintervalo inercial, onde a energia nem é produzida nem dissipada, mas é transferida, por efeito cascata, dos vórtices maiores para os menores. E finalmente a região $C$ é a região de dissipação, onde a energia cinética é convertida em energia interna pela interação molecular.

\subsubsection{O Subintervalo Inercial}

Nesta região os vórtices não sentem os efeitos da dissipação viscosa nem a geração de energia cinética turbulenta. Eles adquirem energia dos vórtices maiores e perdem para os menores, através do efeito "cascata". Assim, para um fluxo turbulento a taxa de decréscimo de energia do espectro tem que equilibrar a taxa de dissipação dos vórtices menores.

Kolmogorov (1941) foi quem primeiro concebeu a idéia deste subintervalo inercial, que separa a região que contém energia da região de dissipação. Argumentos teóricos sugerem que nesta região a turbulência seja isotrópica. Isotropia implica que o campo de velocidades é independente de rotações e reflexões sobre os eixos espaciais. Mesmo que, para os vórtices da região de entrada de energia, a turbulência não possa ser considerada isotrópica, pode-se assumir que em pequenas escalas a estrutura da turbulência é efetivamente isotrópica. Esta isotropia local (refere-se ao número de onda e não ao espaço físico) é importante para a descrição estatística da turbulência em escalas menores. Assim, existindo isotropia local no subintervalo inercial, tem-se a seguinte relação entre os espectros de $u, v$ e $w$ :

$$
F_{v}(k)=F_{w}(k)=\frac{4}{3} F_{u}(k)
$$

Em uma representação log-log dos espectros, a lei de potência -5/3 aparece como inclinação constante e a relação de 4/3 como separação fixa entre as componentes da velocidade. Outra conseqüência da isotropia local é o desaparecimento de todas as correlações cruzadas entre as componentes da velocidade e entre as componentes da velocidade e escalares, o que implica na inexistência de qualquer fluxo turbulento no subintervalo inercial.

Usam-se como teste para verificar a existência do subintervalo inercial estas três condições: a lei de potência de $-5 / 3$, a razão 4/3 entre as componentes ( $u$ e $w$ ) e ( $u$ e $v$ ) do vento e o desaparecimento ou quase nenhuma correlação. A lei de potência $-5 / 3$ estende-se aproximadamente ao número de onda $k=0,1 \eta^{-1}$, a partir do qual, com o aumento de $k$, nitidamente começa a decair (Dubovikov e Tatarskii, 1987).

\subsubsection{Intervalo de Entrada de Energia}

No intervalo de entrada de energia, as formas espectrais tendem a diferenciar-se para cada variável, desde que as escalas integrais correspondentes sejam diferentes. Porém, todas as formas espectrais parecem comportar-se de maneira consistente quando plotados em termos adimensionais semelhantes. Implícito no desenvolvimento de formas espectrais nesta região

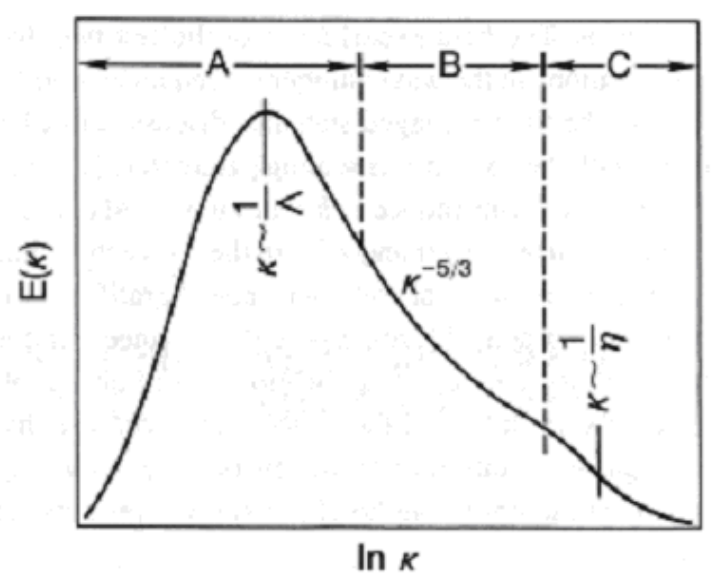

Figura 1 -Visualização do espectro de energia na camada limite mostrando as regiões distintas de entrada de energia (A), subintervalo inercial (B) e a região de dissipação (C). (Fonte: Kaimal e Finnigan, 1994). 
está a suposição da existência de um “gap espectral”, que separa a turbulência da camada limite de flutuações externas a ela. Este “gap espectral” freqüentemente é encontrado para freqüências $f$ entre 0,001 e 0,0001 Hz (Van der Hoven, 1957). Porém, esta extremidade do espectro esta suscetível à influência de tendências em longo prazo presente nos dados. Estas tendências podem ser causadas por ondas gravitacionais, variações diurnas, indução sinóptica ou simplesmente por erros no sensor. Sem a presença de tais tendências nos dados, $\mathrm{F}(k)$ tende a um valor constante quando $k$ tende a zero. Isto é uma conseqüência da representação unidimensional do espectro de energia turbulenta tridimensional.

Nesta representação é difícil identificar os picos espectrais. Para superar esta dificuldade e obter uma representação mais precisa dos picos e vales na distribuição de energia turbulenta, é usual plotar o espectro de energia $k \mathrm{~F}(k)$ e não a densidade espectral de energia. Esta representação do espectro é chamada como espectro logarítmico, pois representa a variância por unidade de intervalo de número de onda. Suas unidades são iguais à variância $\left(\mathrm{m}^{2} \mathrm{~s}^{-2}\right)$ ao invés de variância por intervalo de número de onda $\Delta k$.

\subsection{Espectros sobre Terrenos Planos e Uniformes}

Os experimentos de campo das últimas duas décadas confirmaram que as representações espectrais no espaço dos números de onda ou das freqüências seguem as leis de similaridade em superfícies homogêneas. Quando adimensionalizadas com os parâmetros de escala apropriados $\left(u_{*}, T_{*}, W_{*}\right)$ as formas espectrais se reduzem a uma família de curvas universais, que são funções somente de $z / L$ (onde $L$ é o comprimento de Obukhov) na camada superficial e $z / z_{i}$ (onde $z_{i}$ é a altura da camada) em condições convectivas. Em um nível mais prático, isso possibilita fazer-se o uso dessas equações, submetendo-as a várias aplicações, desde o desenho de estruturas como, por exemplo, pontes, prédios, foguetes e até mesmo no desenvolvimento de modelos de poluição do ar.

\subsection{Aliasing}

Uma regra básica na análise de dados é que pelo menos 2 pontos do conjunto de dados são requeridos por período ou por comprimento de onda para resolver uma onda. A partir da análise de Fourier envolvendo divisões arbitrárias nos sinais dentro das ondas, os dois pontos de dados requeridos também asseguram o sinal como arbitrário. Por exemplo, se existe um total de $\mathrm{N}$ pontos, então a maior freqüência, ou menor comprimento de onda, que pode ser resolvida numa Transformada de Fourier é $n_{f}=N / 2$, a qual é chamada de Freqüência de Nyquist.

O que acontece então com o sinal físico de alta freqüência que não é medido ou digitalizado com freqüência suficiente para resolver o sinal? A resposta é que o sinal verdadeiro de alta freqüência é rebatido dentro de uma freqüência menor, criando um erro e iludindo a transformada de Fourier.

Se existe uma freqüência $n_{h}$ que é maior do que a Freqüência de Nyquist, então o sinal ou amplitude daquela onda será rebatido numa determinada freqüência dada por $n-N-n_{h}$, onde ela será adicionada a alguma verdadeira amplitude, já existente em $n$. O problema de aliasing pode existir em qualquer série de dados e ocorre em duas situações: na qual a taxa de amostragem é superior a capacidade de medição do sensor dado pelo tempo de resposta e o processo físico tem freqüência maior do que a taxa de medida (Stull, 1988).

\subsection{Removendo as Tendências}

A presença de uma tendência em uma série temporal faz com que os dados se tornem não estacionários e, portanto menos apropriados para a análise. Pode-se definir uma tendência como qualquer componente de freqüência com um período maior do que o comprimento de gravação. Nos limites dos períodos muito longos essa tendência pode parecer ser linear dentro do período. O método de mínimos quadrados é freqüentemente usado para remover as tendências lineares e polinomiais. Para muitas aplicações, o filtro de passa alta digital é preferido porque é mais simples e é mais bem entendido. O mesmo filtro digital deve ser aplicado a todas as variáveis processadas, assegurando o tratamento uniforme de baixas freqüências para todo o sinal.

Tendências numa série temporal produzem distorções no final das freqüências baixas do espectro. Esta distorção, se suficientemente grande, pode mascarar totalmente o verdadeiro máximo do espectro e fazer com que o espectro continue ascendendo com o decréscimo da freqüência.

A remoção da tendência não garante que a verdadeira forma do espectro pode ser obtida. O método particular de remoção de tendências usado freqüentemente determina a forma do espectro sem tendências e a localização do seu máximo. Remover tendências deveria ser realizado, somente se essas tendências forem fisicamente esperadas ou claramente aparentes nas séries temporais. Remoções de tendências automáticas não são recomendadas, exceto para certas variâncias e cálculos de fluxos aonde a presença de tendências podem ser altamente prejudiciais.

Remover as tendências nos dados significa subtrair do grupo de dados escolhido uma linha reta que melhor se ajuste àquele segmento de dados. Em geral qualquer baixa freqüência com um período maior do que todo o período de medidas, irá também gerar o ruído vermelho. Se o período desta freqüência é conhecido, então se pode ajustar a curva através do teste padrão de mínimos quadrados e subtrair este resultado da série principal. 
Depois de realizada essa remoção de tendências, as formas pontiagudas da janela de dados causam o que é chamado de espalhamento, onde as estimativas espectrais, a partir de qualquer freqüência, são contaminadas com algumas amplitudes escapando das freqüências vizinhas. Para reduzir o escapamento destas freqüências, recomendam-se janelas de dados modificadas com bordas lisas (Stull, 1988).

\section{LOCALIZAÇÃO DA REGIÃO DE ESTUDO, DESCRIÇÃO DOS DADOS E CLIMATOLOGIA DO LOCAL}

O Centro de Lançamento de Alcântara está localizado no município de Alcântara no litoral norte do Estado do Maranhão, com coordenadas geográfica de $2^{\circ} 19^{\prime} S$ de latitud e $44^{\circ} 22^{\prime} \mathrm{W}$ de longitude e está a $40 \mathrm{~m}$ de altitude, distante $30 \mathrm{~km}$ em linha reta da cidade de São Luiz. Desse centro são lançados os foguetes brasileiros, tais como o Veículo Lançador de Satélite (VLS) e os de Sondagens (SONDA II, SONDA III, VSB30 e VS40). A vegetação deste local é característica de região de restinga, com altura média em torno de 2 a $3 m$, sendo bem fechada do ponto de vista da arquitetura das plantas e o dossel homogêneo em termos de altura. O clima apresenta um regime de precipitação dividido em dois períodos: chuvas intensas durante os meses de janeiro a junho, sendo os meses de março e abril os mais chuvosos, com total mensal superior a $300 \mathrm{~mm}$; e período seco, de julho a dezembro, com precipitação inferior a $15 \mathrm{~mm}$ por mês (Fisch, 1999). Os ventos possuem comportamento distinto

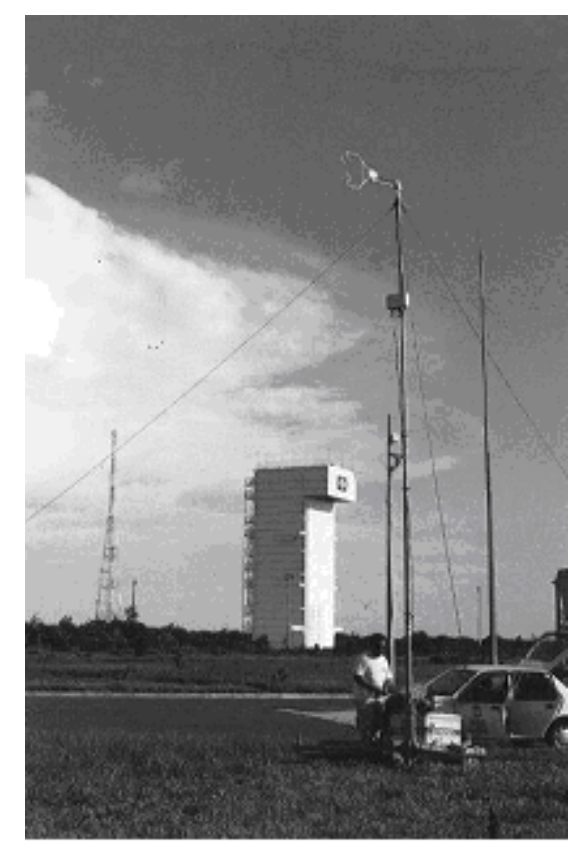

entre a época chuvosa e a seca. Durante a estação chuvosa (mês característico: março) o vento é mais fraco, pois é basicamente devido aos ventos alísios. O vento predominante é de leste até aproximadamente $5.000 \mathrm{~m}$ de altitude, com velocidades em torno de 7 a $8 \mathrm{~m} / \mathrm{s}$ nos níveis entre 1.000 e $3.000 \mathrm{~m}$. Na estação seca (mês de setembro) há uma superposição dos ventos alísios com o fenômeno de brisa marítima, provocando ventos fortes (Fisch, 1999). O vento é predominantemente de leste até aproximadamente $8.000 \mathrm{~m}$ de altitude, com velocidades em torno de 7 a $9 \mathrm{~m} / \mathrm{s}$. O vento é bem intenso na camada mais baixa (até $2.000 \mathrm{~m}$ ), com velocidades máximas entre 10 e $15 \mathrm{~m} / \mathrm{s}$, apresentando uma pequena rotação para sudeste. Isto se deve à intensificação do fenômeno de brisa marítima, que possui sua influência máxima (contraste térmico oceano-continente) durante esta época, em particular nos meses de setembro a novembro. A temperatura do ar e a umidade relativa não apresentam variações sazonais, e seus valores são típicos da atmosfera tropical.

\subsection{Mastro e Sensor Sônico}

Foi instalada uma estrutura metálica de $15 \mathrm{~m}$ de altura, na qual foi colocado o sensor sônico tridimensional a $9 \mathrm{~m}$. O CSAT3D (Campbell Scientific, USA) é um anemômetro sônico tridimensional, que mede a velocidade do vento sobre 3 eixos não-ortogonais (Figura 2). A partir dessas medidas, as componentes ortogonais do vento $(u, v, w)$ e a temperatura $(T)$ foram calculadas.

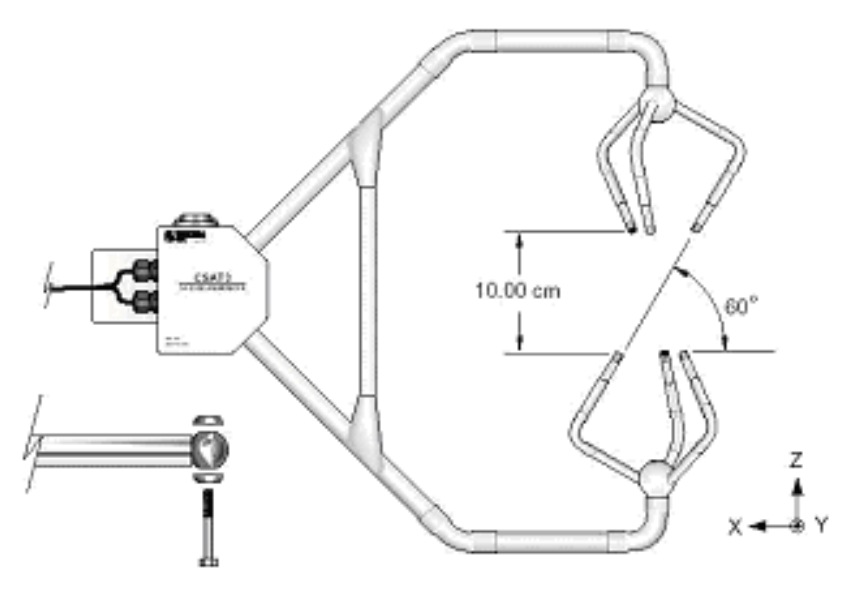

Figura 2 - No lado esquerdo (a) uma imagem do anemômetro sônico preso à torre, no campo. No lado direito (b) uma figura do Anemômetro Sônico Tridimensional (CSAT3D). 


\subsection{Programa em linguagem Fortran}

Os dados brutos deste experimento foram arquivos com medidas de resposta rápida $(20 \mathrm{~Hz})$ das 3 componentes do vento e da temperatura do ar, que foram agrupados em séries diárias de dados, distribuídos em 7 colunas, a saber: Código de identificação; Ano; Dia Juliano; Hora e minuto local; Segundos; Componente $u$, Componente $v$, Componente $w$ e Temperatura. A temperatura do ar foi calculada pelo anemômetro sônico 3D com base na teoria da dependência da velocidade do som a temperatura do ar (Campbell Scientific, 1998). A campanha foi realizada entre os dias 218 a 239 do Calendário Juliano, ou seja, de 05/08/1999 a 26/08/1999. Este período compreende a época seca da região do CLA.

Foi desenvolvido um programa usando a linguagem computacional Fortran, para a análise dos dados experimentais. Nele foram feitas as rotações de eixo propostas por Kaimal e Finnigan (1994), para garantir que as componentes meridional e vertical do vento médio fossem nulas. O programa calcula também os valores médios das variáveis vento e temperatura $(U, T)$, as variâncias, os fluxos de momentum e de calor e, por fim, os espectros de freqüência das componentes do vento. A seguir foi usado um programa de médias espectrais que divide todo o espectro em 22 bandas. Estas bandas ou blocos são definidos a partir das freqüências mínima e máxima do espectro, sendo que as subdivisões das demais bandas intermediárias são realizadas dentro da escala logarítmica (Kaimal e Gaynor, 1983).

\section{RESULTADOS}

\subsection{Cálculo dos Espectros de Freqüência}

Os espectros foram determinados a partir das séries temporais de $32768\left(2^{15}\right)$ pontos de dados coletados a uma taxa de $20 \mathrm{~Hz}$. O intervalo de tempo usado foi de 30 minutos aproximadamente, que é o tempo médio usado em estudos de determinação de fluxo (Lee et al., 2004). Os espectros calculados foram então classificados conforme a estabilidade atmosférica. Devido à representatividade da quantidade de pontos envolvidos em cada conjunto de classe de estabilidade, optou-se por separar em apenas 3 grupos de estabilidade para desenvolver a análise, mas que compreendem $93 \%$ da quantidade total de dados, a saber: duas classes representativas do período convectivo: $-0,3<\mathrm{z} / \mathrm{L}<-0,1$ e $-0,1<\mathrm{z} / \mathrm{L}<0$ com 48.598 e 69.938 pontos, respectivamente e uma classe no período estável $0<\mathrm{z} / \mathrm{L}<0,1 \mathrm{com}$ 164.208 pontos. O espectro médio foi então obtido, através do esquema de média de blocos.

Para cada espectro individual, a taxa de dissipação de Energia Cinética Turbulenta representada por $\left(\Phi_{\varepsilon}\right)^{2 / 3}$ foi determinada a partir da expressão de Kolmogorov para o subintervalo inercial:

$$
\frac{n S_{i}}{u_{*}^{2}}=\frac{\alpha_{i}}{(2 \pi \kappa)^{2 / 3}} \phi_{\varepsilon_{i}}^{2 / 3} f^{-2 / 3}
$$

onde $n$ é a freqüência em $\mathrm{Hz}, S_{i}$ a densidade espectral, $u_{*}$ a velocidade de fricção, $k$ a constante de von-Kárman, $\Phi_{\varepsilon}=\kappa z$ $\varepsilon /\left(u_{*}\right)^{3}$ a razão de dissipação adimensional, $f=n z / U$ a freqüência adimensional e $\alpha_{i}(i=u, v, w)$ a constante de Kolmogorov. Incluindo-se $\left(\phi_{\varepsilon}\right)^{2 / 3}$ na normalização dos espectros $u, v$ e $w$ remove-se a dependência das mesmas em $z / L$ (Kaimal et al., 1972). Este procedimento faz com que os espectros sejam coincidentes no subintervalo inercial.

Os espectros médios foram representados por expressões matemáticas clássicas propostas na literatura (Sorbjan, 1989), sto é:

$$
\frac{n S_{i}}{u_{*}^{2} \phi_{\varepsilon}^{2 / 3}}=\frac{A f}{(1+B f)^{2 / 3}}
$$

no qual $A$ e $B$ são coeficientes empíricos e determinados localmente. As Figuras 3, 4 e 5 apresentam os espectros de freqüência das componentes do vento $(u, v, w)$ para as 3 classes de estabilidade que estão sendo analisadas.

Em todas as figuras, verifica-se a existência de uma região na qual a lei de -2/3 de Kolmogorov é obedecida, isto é, a existência de uma região na qual a turbulência possui um regime de isotropia. O início da região de subintervalo inercial para as velocidades $v$ e $w$ é em torno de $f \sim 1$, ao passo que para a velocidade longitudinal $u$ observou-se este inicio em torno de $f \sim 0,2$, para todas as classes de estabilidade.

Observou-se também, que a determinação do pico espectral para a velocidade vertical é mais evidente do que para ambas as velocidades horizontais. As freqüências máximas da velocidade vertical associadas com os máximos espectrais, tiveram seus valores entre 0,5 e 0,6 para as 3 classes de estabilidade aqui observadas. Estes valores são inferiores àqueles observados no experimento de Kansas (Kaimal et al., 1972), mas são muito parecidos com os valores observados no experimento de uma superfície complexa no município de Dona Francisca, RS (Magnago et al., 2009).

Para as velocidades horizontais $u$ e $v$ na região de baixas freqüências, notou-se a existência de uma freqüência de corte entre $\sim 0,02$ e $\sim 0,03$, como sendo o valor de início desta frequiência para todos os casos (Figuras 3 e 4). Quando as freqüências estão localizadas abaixo da freqüência crítica, 
todos os espectros da velocidade lateral $v$ mostram um claro crescimento com o decréscimo da freqüência (Figura 4). Isso ocorre devido ao intervalo de tempo considerado ( 30 minutos) contaminar a determinação dos fluxos computados por capturar movimentos de mesoescala (por exemplo, a influência do ciclo diurno do vento associado ao fenômeno de brisa marítimica local).

Na região de altas freqüências em que ocorre o fenômeno de aliasing, para o caso das velocidades longitudinais $u$, a freqüência de início do fenômeno é dada por 5 (Figura 3) e para os casos das velocidades laterais v esse início foi de 10 (Figura 4), para as 3 classes de estabilidade usadas. Este fenômeno ocorre com menor intensidade para a componente $w$.

Destes espectros anteriormente citados, também se obteve os coeficientes $A$ e $B$ que melhor ajustam a curva espectral. Estes valores são obtidos a partir da Equação 4. Notase que os valores para a componente $u$ são bem maiores do que os das outras duas componentes ( $v$ e $w$ ), em todas as classes de estabilidade. Isto se deve, em parte, ao fato de que o vento horizontal é bem maior do que o vertical e muito persistente, o que faz com que a flutuação lateral seja pequena.

Na Figura 6 são apresentadas as flutuações das velocidades horizontais $\left(\sigma_{u}, \sigma_{v}\right)$, normalizados pela velocidade de fricção $\left(u_{*}\right)$, sendo semelhante àquelas obtida por Leavitt e Paulson (1975), embora o espalhamento vertical dos pontos para o terreno analisado não foi observado. Já para a velocidade vertical $w$ o gráfico de $\sigma_{w} / u_{*}$ está muito parecido com o mostrado por eles em 1975. Em relação à dependência destas flutuações com a estabilidade atmosférica, praticamente $\sigma_{w}$ não depende
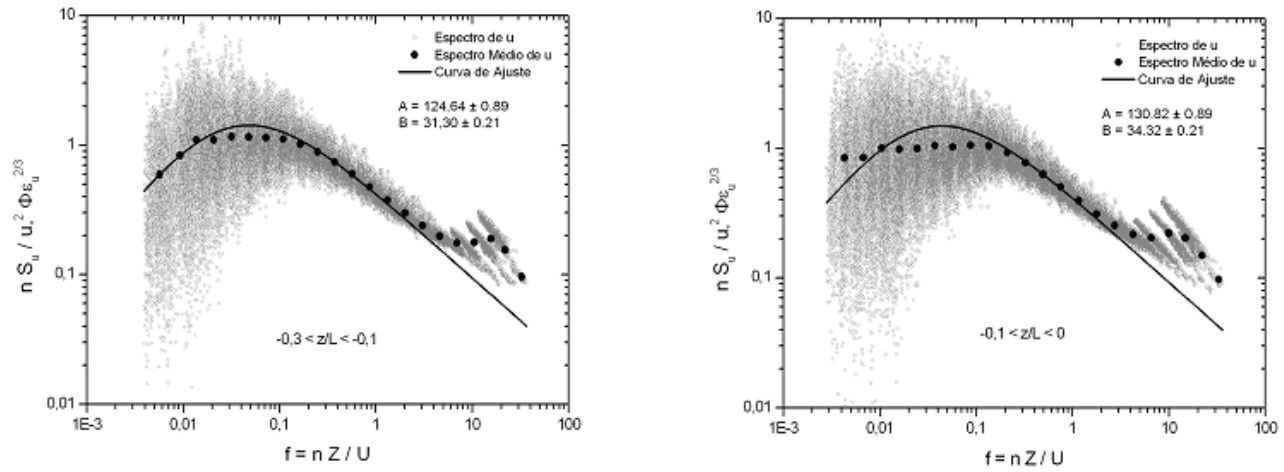

Figura 3 - Espectro da velocidade horizontal para as 3 classes de estabilidade. Os pontos cinza pequenos representam o espectro bruto. Os círculos pretos maiores representam o espectro médio em suas 22 bandas. A linha contínua representa a curva de ajuste proposta pela Equação 4.
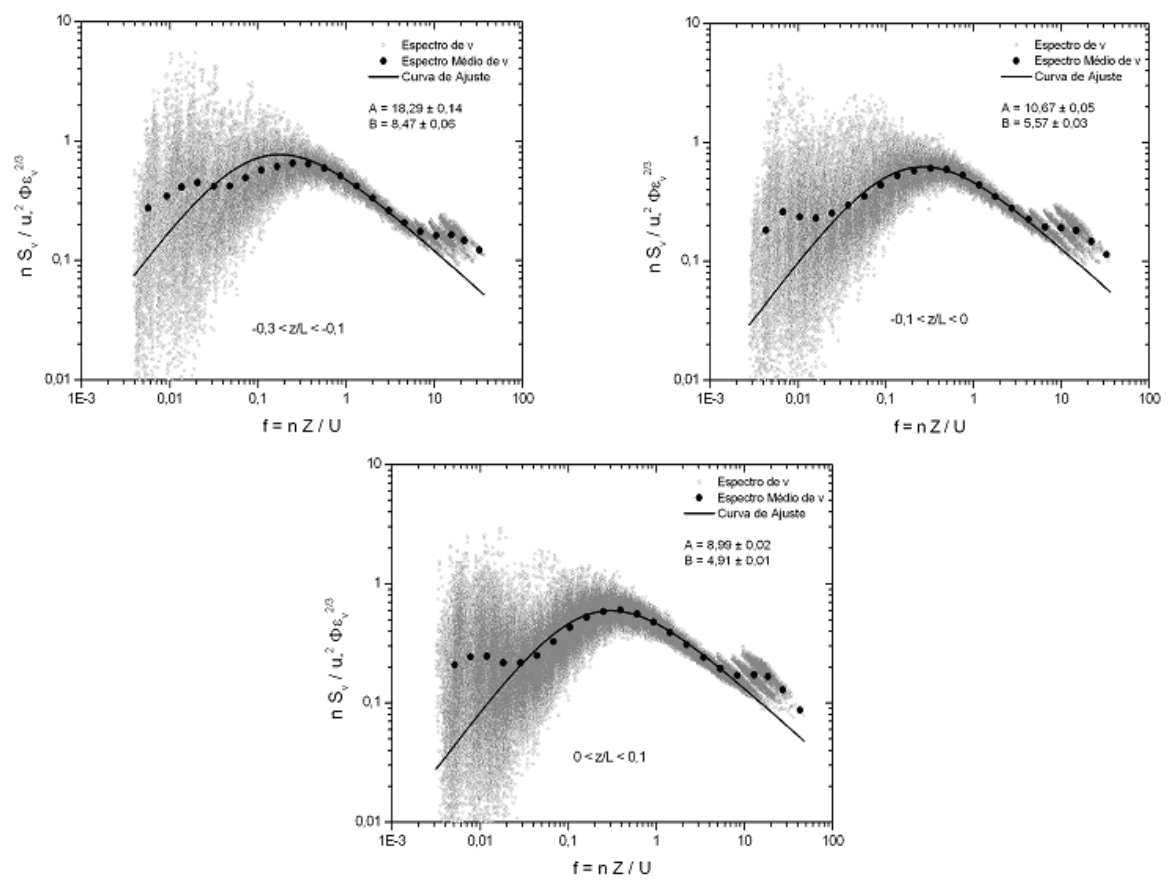

Figura 4 - Espectro da velocidade lateral $v$ para as 3 classes de estabilidade. Os pontos cinza pequenos representam o espectro bruto. Os círculos pretos maiores representam o espectro médio em suas 22 bandas. A linha contínua representa a curva de ajuste proposta pela Equação 4. 
da estabilidade atmosférica, ou seja, é um valor constante em torno de 1,3. Isto faz sentido, pois a estabilidade atmosférica é próxima da neutralidade. A flutuação de $\sigma_{u} / u_{*}$ é constante para valores negativos de $z / L$ e levemente linear para valores positivos de $z / L$. A flutuação de $\sigma_{u} / u_{*}$ possui uma dependência da estabilidade.

Moraes (2000) obteve sobre regime quase-neutro na região dos pampas gaúchos, valores para $\sigma_{u} / u_{*} \sim 2,42$ e valores para $\sigma_{v} / u_{*} \sim 2,78$. Monin e Yaglow (1971) sugeriram valores iguais à $\sigma_{u} / u_{*} \sim 2,3$ e $\sigma_{v} / u_{*} \sim 1,7$ sob estratificação neutra. Kaimal et al. (1972) encontraram valores como $\sigma_{u} / u_{*} \sim 1,8$ e $\sigma_{v} / u_{*} \sim 1,6$, mas em virtude da falta de similaridade entre os espectros dos diferentes locais de observação, existe uma pequena razão para esperar $\sigma_{u} / u_{*}$ e $\sigma_{v} / u_{*}$ como sendo funções universais. Finalmente, Arya (2001) faz uma revisão de literatura destes valores e sugere que, para condições próximas a neutralidade, estes valores de $\sigma_{u} / u_{*}, \sigma_{v} / u_{*}$ e $\sigma_{w} / u_{*}$ deveriam ser iguais a 2,4, 1,9 e 1,3, respectivamente. Os valores médios aqui calculados para $\sigma_{u} / u_{*}, \sigma_{v} / u_{*}$ e $\sigma_{w} / u_{*}$ são $2,32,1,87$ e 1,32 respectivamente, concordam muito bem com os valores determinados experimentalmente em outros sítios citados acima.

\subsection{Determinação dos Parâmetros Micrometeorológicos}

Com o objetivo de descrever as características turbulentas do local, calculou-se para cada classe de estabilidade, os parâmetros meteorológicos: velocidade de fricção $\left(u_{*}\right)$ e comprimento de rugosidade aerodinâmico $\left(\mathrm{Z}_{\mathrm{o}}\right)$. Além disso, determinaram-se os valores de $T_{*}$ para se ter uma idéia desta escala de temperatura, bem como, os valores médios do vento $(U)$. Todas estas informações estão apresentadas na Tabela 1.

Tabela 1 - Parâmetros Micrometeorológicos determinados em função de classes de estabilidade.

\begin{tabular}{|c|c|c|c|c|}
\hline 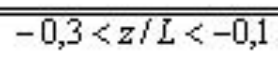 & Média & Desvio Padrão & Mínimo & Máximo \\
\hline$u(\mathrm{~m} / \mathrm{s})$ & 0,49 & 0,07 & 0,28 & 0,73 \\
\hline T. $\left({ }^{\circ} \mathrm{C}\right)$ & 0,38 & 0,11 & 0,09 & 0,68 \\
\hline$U(\mathrm{~m} / \mathrm{s})$ & 4,76 & 0,93 & 2,26 & 7,04 \\
\hline$Z_{n}(\mathrm{~m})$ & 0,22 & 0,12 & 0,007 & 0,85 \\
\hline$-0,1<z / L<0$ & Média & Desvio Padrão & Mínimo & Máximo \\
\hline$u$ & 0,59 & 0,12 & 0,29 & 0,76 \\
\hline$T$ & 0,17 & 0,11 & 8 & 0,5 \\
\hline$U$ & 5,96 & 1,47 & 2,27 & 7,47 \\
\hline$Z_{0}$ & 0,18 & 0,10 & 0,01 & 0,67 \\
\hline $0<<z / I<0,1$ & Média & Desvio Padrão & Mínimo & Máximo \\
\hline$u$ & 0,48 & 0,10 & 0,18 & 0,9 \\
\hline$T$ & $-0,04$ & 0,01 & $-0,09$ & Q \\
\hline $\bar{U}$ & 4,95 & 1,03 & 1,74 & 8,63 \\
\hline$Z$ & 0,15 & 0,06 & 0,009 & 0,44 \\
\hline
\end{tabular}



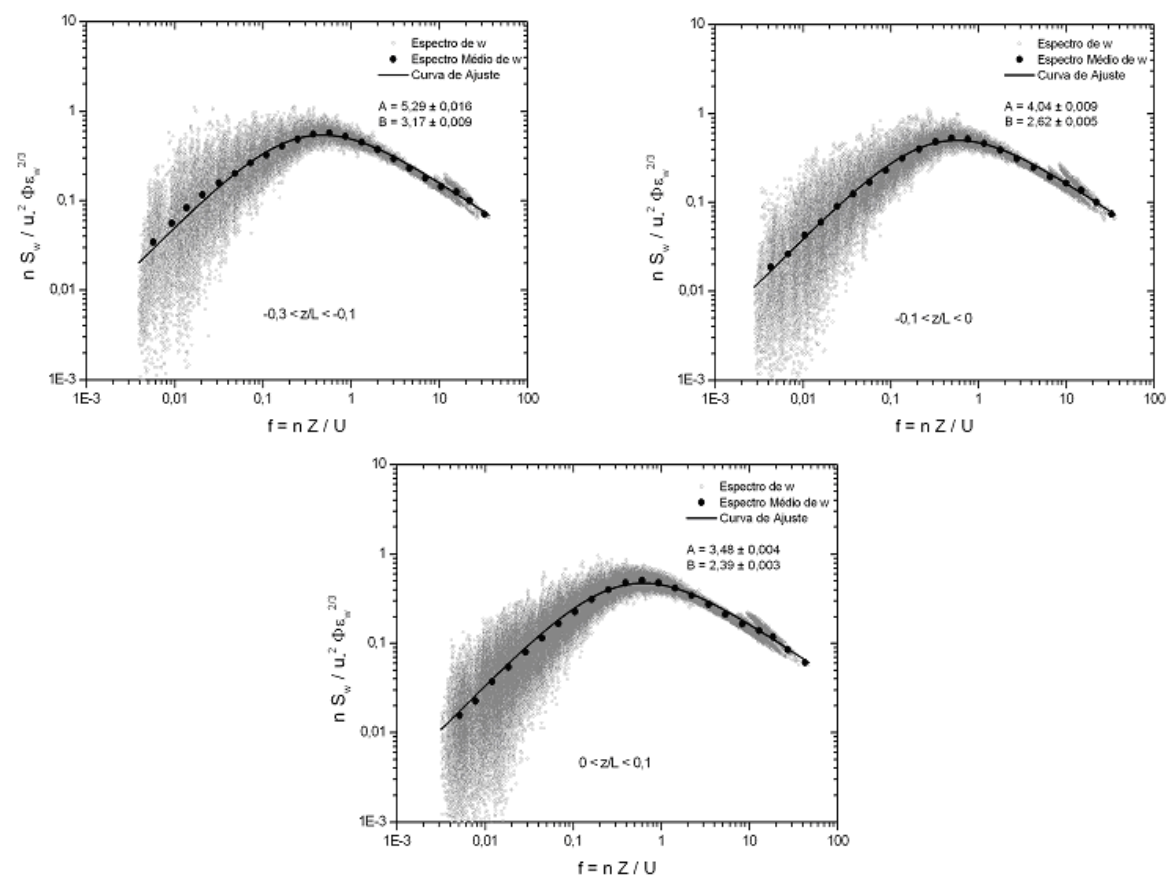

Figura 5 - Espectro da velocidade vertical $w$ para as 3 classes de estabilidade. Os pontos cinza pequenos representam o espectro bruto. Os círculos pretos maiores representam o espectro médio em suas 22 bandas. A linha contínua representa a curva de ajuste proposta pela Equação 4.

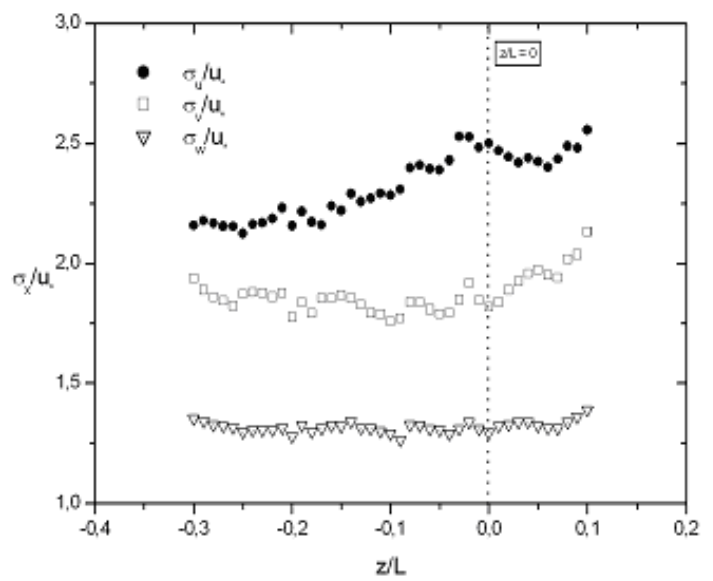

Figura 6 - Razão entre os espectros médios das velocidades: longitudinal, lateral e vertical adimensionalizadas pela velocidade de fricção plotadas contra o parâmetro de estabilidade .

Tabela 2 - Relações $u / U$ e $u / Z$ para as classes de estabilidade.

\begin{tabular}{||c|c|c|}
\hline Classes de Estabilidade & $u / U$ & $u / Z$ \\
\hline \hline$-0,3<z / L<-0,1$ & 0,103 & 2,23 \\
\hline$-0,1<z / L<0$ & 0,099 & 3,28 \\
\hline $0<z / L<0,1$ & 0,097 & 3,20 \\
\hline
\end{tabular}

Roballo e Fisch (2008), utilizando-se de dados de perfil de vento em uma torre anemométrica instalada próximo ao local desta coleta de dados, obtiveram valores médios para o mês de agosto (mês que foi realizada a campanha intensiva com o sônico $3 \mathrm{D})$ de $u_{*=} 0,42 \mathrm{~m} / \mathrm{s}, U=4,9 \mathrm{~m} / \mathrm{s}$ e $Z_{o}=0,25 \mathrm{~m}$. Ressalta-se que estes valores são derivados de um conjunto de dados independente dos utilizados neste trabalho. Isto é um indício da robustez dos resultados em caracterizar a turbulência atmosférica do local

Foram também calculados valores para as relações $U_{*} / U$ e $u_{*} / Z_{o}$ para todas as classes de estabilidade aqui estudadas, buscando uma associação entre estes parâmetros e as diferentes classes de estabilidade atmosférica (Tabela 2). Observa-se que é bem constante a relação adimensional entre $U$ em torno de 0,1 .

\section{CONCLUSÕES}

Este trabalho objetivou a determinação do espectro de freqüência das componentes do vento na região do CLA, para aplicação em estudos de P\&D de Meteorologia Aeroespacial. A partir de um conjunto de dados de anemometria sônica (dados de resposta rápida) coletados durante o período seco (agosto), determinaram-se os espectros observacionais, bem como suas curvas de ajuste. $\mathrm{O}$ envelope para a componente $u$ é bem superior ao das componentes $v$ e $w$, devido ao fato dos ventos na região serem bem intensos e persistentes em uma única direção. A partir destes dados, também se determinou 
parâmetros micrometeorológicos que caracterizam a turbulência local (por exemplo, $u_{*}, Z_{o}, T_{*}$ ), os quais são próximos aos determinados neste mesmo local por uma outra técnica (o uso de dados de uma torre anemométrica ajustando-se o perfil logarítmico do vento). Isto mostra a robustez dos resultados alcançados. Finalizando, pode-se concluir que, no período de seca da região do CLA, os ventos são tão intensos que acabam provocando a mistura turbulenta local e a geração de turbulência acaba sendo puramente mecânica. Portanto, pode-se assumir que não há um ciclo diurno característico da estabilidade atmosférica, no qual o período diurno seja de caráter convectivo e o período noturno seja de características estáveis. Os resultados encontrados e a semelhança entre eles sugerem que o escoamento atmosférico tenha, durante todo o dia, características de quase neutralidade.

\section{AGRADECIMENTOS}

Os autores agradecem ao CNPq pela bolsa de Pós Doutorado Júnior, concedida ao autor Roberto Magnago, no período de Fevereiro de 2008 até Fevereiro de 2009.

\section{REFERÊNCIAS BIBLIOGRÁFICAS}

ARYA, S. P. Introduction to Micrometeorology (second edition). Academic Press, Orlando, United States, 420 p., 2001

BARBOSA, R. L; OYAMA, M. D.; MACHADO, L. A. Climatologia das perturbações convectivas iniciadas na costa norte do Brasil. Revista Brasileira de Meteorologia. v. 21, n. 1, pp. 107-117, 2006.

CAMPBELL SCIENTIFIC - CSAT3 TRIDIMENSIONAL SONIC ANEMOMETER, MANUAL, 50 p., 1998. (http:// www.campbellsci.com/documents/manuals/csat3.pdf)

COUTINHO, E. C.; FISCH, G. Distúrbios ondulatórios de leste (DOLs) na região do Centro de Lançamento de Alcântara - MA. Revista Brasileira de Meteorologia. v.22, n.2, p.193-203, 2007.

DUBOVIKOV, M. M.; TATARSKII, V. I. Models Of The Scalar Spectrum For Turbulent Advection. Fluid Mech. 88. 641-52. 1987.

FISCH, G. Características do Perfil Vertical do Vento no Centro de Lançamento de Foguetes de Alcântara (CLA). Revista Brasileira de Meteorologia, v. 14, n.1, p. 11-22, 1999.

KAIMAL, J. C.; WYNGAARD, J. C.; IZUMI, Y.; COTÉ, O. R. Spectral Characteristics Of Surface-Layer Turbulence. Quart. Journal R. Meteorological Society, 98, 563-589. 1972.
KAIMAL, J. C., GAYNOR. The Boulder Atmospheric Observatory. J. Clim. Appl. Meteorol. v.22, 863-880. 1983.

KAIMAL, J. C.; FINNIGAN J. J., Atmospheric Boundary Layer Flows - Their Structure and Measurement, Oxford University Press. New York. 298 pp. 1994.

KOLMOGOROV, A.N. Dissipation Of Energy In A Locally Isotropic Turbulence. Doklady Akad. Nauk SSSR, 32, 141. 1941.

LEAVITT, E.; PAULSON, C.A. Statistics os Surface Layer Turbulence Over The Tropical Ocean. J. Phys. Oceanogr. v.5: 143-156. 1975.

LEE, X.; MASSMAN, W.; LAW, B. Handbook Of Micrometeorology - A Guide for Surface Flux Measurement. Kluwer Academic Publishers. 250pp. 2004.

MONIN A.S.; YAGLOW A.M. Statiscal Fluid Mechanics, Vols $1 \&$ 2. , John Lumley (ed.), The MIT Press, Cambridge, 769pp. 1971.

MAGNAGO R.; MORAES, O. L. L.; ACEVEDO, O.C.; Turbulence Velocity Spectra Dependence On The Mean Wind At The Bottom Of A Valley. Phisica A. 2009. Volume 388, Edição: 9, Maio 2009, Pags 1908-1916.

MORAES, O. L. L. Turbulence Characteristics In The Surface Boundary Layer Over The South American Pampa. Boundary-Layer Meteorology. v. 96: 317-335. 2000.

NIEUWSTADT, F. T. M.; VAN DOPP, H. Atmospheric Turbulence And Air Pollution Modeling. Boston, D. Reidel Publishing Company, 355pp. 1981.

PIRES, L. B. M., FISCH, G., SOUZA, L. F., AVELAR, A. C., GIRARDI, R. M., A study of the Internal Boundary Layer and turbulence generated at the Alcântara Launching Center, Boundary Layer Meteorology. [submetido], 2010.

ROBALLO, S.; FISCH, G. Escoamento Atmosférico no Centro de Lançamento de Alcântara (CLA): Parte I - Aspectos Observacionais. Revista Brasileira de Meteorologia, v.23, n.4, 510 - 518, 2008.

SORBJAN, Z. Structure Of The Atmospheric Boundary Layer.

Prentice Hall, Englewood Cliffs, NJ, 317 pp. 1989.

STULL, R. B. An Introduction to Boundary Layer Meteorology.

Kluwer Academic Press, Dordrecht, The Netherlands, 666 pp. 1988.

VAN DER HOVEN, I. Power Spectrum Of Horizontal Wind Speed In The Frequency Range From 0,0007 To 900 Cycles Per Hour. J. of Met. 14, 160-164. 1957. 